\section{Comparison of electrocautery and plasmablade on ischemia and seroma formation after modified radical mastectomy for locally advanced breast cancer}

\author{
Husnu Alptekin, ${ }^{1}$ Huseyin Yilmaz, ${ }^{1}$ \\ Bahadir Ozturk, ${ }^{2}$ Ilhan Ece, ${ }^{1}$ \\ Mehmet Ertugrul Kafali, ${ }^{1}$ \\ Fahrettin Acar ${ }^{1}$ \\ ${ }^{1}$ Department of Surgery, Faculty of \\ Medicine, Selcuk University, Konya; \\ ${ }^{2}$ Department of Biochemistry, Faculty of \\ Medicine, Selcuk University, Konya, \\ Turkey
}

\begin{abstract}
The aim of this study was to compare postoperative drainage volumes and IMA levels in patients who underwent modified radical mastectomy (MRM) with using PlasmaBlade (PB) or electrocautery (EC). A total of 36 patients who underwent MRM with PB or EC in our clinic between August 2012 to February 2013 were enrolled. Number of removed and positive lymph nodes, duration of drainage and total drainage volume was recorded. Seroma formation after drain removal and number of aspirations were also recorded. Serum ischemia modified albümine (IMA) levels were analysed before surgery, 1 hour and 24 hour after surgery. In total, 36 patients were treated with MRM in the study period. Of the 36 patients, 16 underwent MRM with $\mathrm{PB}$, and 20 underwent MRM with EC. The patients demographics were similar in both groups. The mean drainage volume and seroma formation were significantly higher in the PB group when compared with EC group $(\mathrm{P}<0.05)$. Number of aspirations due to the seroma were also high in PB group. The total aspiration volume of seroma was not different in both groups. IMA levels 24 hours after surgery in the PB group was significantly higher than EC group. There was no statistical significance between the groups for IMA levels at $1^{\text {st }}$ hour. PB is a monopolar energy device and is associated with increased levels of ischemia. This situation resulted with an increased volume of total axillary drainage and elevated risk of seroma formation.
\end{abstract}

\section{Introduction}

Worldwide, it is estimated that more than one million women are diagnosed with breast cancer every year, and more than 410,000 will die from the disease and it is the second leading cause of cancer death among women. ${ }^{1}$

Surgery is considered the main treatment option for breast cancer and seroma is one of the most common complication developing after surgical therapy. At the postoperative period, suction drains is routinely used to remove the fluid accumulation in the axillary space. Nevertheless, prior to the appropriate reduction in drainage volume removal of drains may result in seroma formation. Seroma was defined as a serous fluid collection that develops under the skin flaps or in the axillary dead space after axillary dissection. ${ }^{2}$ Seroma formation can be seen more than $50 \%$ in patients with axillary lymph node dissection. ${ }^{3-6}$ Although seroma is not life threatening complication, it can lead to significant morbidity (e.g. flap necrosis, wound dehiscence, predisposes to sepsis, prolonged recovery period). However, formation of seroma does not lead to delay of adjuvant therapy. ${ }^{7}$ Fluid collection can be managed in several weeks, by repeated needle aspiration to seal the skin flaps against the chest wall. ${ }^{8}$

Age, tumor size, surgical technique, the use of electrocautery (EC), preoperative chemotherapy and many other factors may affect the seroma formation. ${ }^{9-11}$ It is claimed that, the use of EC increases seroma formation due to the tissue ischemia. ${ }^{12}$ PEAK PlasmaBlade (PB) (Peak surgical inc. CA,USA), is an electrosurgical device that uses radiofrequency energy in short pulses via an insulated cutting electrode, and creates an effective cutting edge while the blade stays near body temperature. This feature of the device is presented as a factor which reduces tissue ischemia. ${ }^{13}$ Ischemia-modified albumin (IMA) has emerged as a sensitive biomarker in the evaluation of tissue ischemia, and was used in many ischemic conditions such as myocardial ischemia, skeletal muscle ischemia, pulmonary embolism, and stroke. ${ }^{13-16}$ To the best of our knowledge, there are no studies showing whether this increases after axillary dissections during mastectomy.

In this study; we aimed to evaluate postoperative drainage volumes and IMA levels at patients who underwent modified radical mastectomy (MRM) with using $\mathrm{PB}$ or EC.
Correspondence: Ilhan Ece, Department of Surgery, Faculty of Medicine, Selcuk University, Konya 42075, Turkey.

Tel: +903322415000-44713; Fax: +903322412184

E-mail: ilhanece@yahoo.com

Key words: Ischemia modified albumin; Breast; Cancer; Surgery; Morbidity.

Conflict of interest: the authors declare no potential conflict of interest.

Received for publication: 18 December 2016 Revision received: 27 February 2017.

Accepted for publication: 28 February 2017.

This work is licensed under a Creative Commons Attribution NonCommercial 4.0 License (CC BY-NC 4.0).

C Copyright H. Alptekin et al., 2017

Licensee PAGEPress, Italy

Surgical Techniques Development 2017; 7:7011 doi:10.4081/std.2017.7011

\section{Materials and Methods}

Between August 2012 to February 2013, consecutive patients who underwent MRM for unilateral, locally advanced primary breast cancer (T2 or T3) were included in the study. The study was approved by the local ethical committee. Signed and informed consent was obtained from all patients by the responsible surgeon. The study was designed as non-randomised prospective study. The type of procedure to be performed was selected according to surgeons choice. The exclusion criteria were as follows: Patients with early breast cancer, history of previous breast or axillary surgery, uncompensated diabetes, advanced liver disease, severe obesity and history of neoadjuvant chemotherapy. Patients who underwent breast reconstruction were not included in the study.

Following medical evaluation, the patients were admitted to the hospital on the day of surgery. Operations were performed by the same surgical team in all cases. Two type electromechanical devices were used during surgery to reduce blood loss and operating time. In 20 of patients, preparations of skin flaps, and excision of the all breast tissue was performed with EC (Valley $\mathrm{Lab}^{\mathrm{TM}}$ diathermy power of 50 watt). Only those vessels larger than $5-\mathrm{mm}$ in diameter were ligated with suture tie in axillary region. In 16 of patients, dissection was performed with PB. Two closed suction drains were placed in the dissection area. One was placed in the axilla and the other was laid under inferior skin flap. At the end of the 
operation, all wounds were covered with dry and sterile gauze. The pressure dressing consisted of a circumferential wrap using two $15 \mathrm{~cm}$ elastic bandages. The same surgical technician applied the dressing to ensure the equal degree of pressure in each patient. The pressure dressing remained in place until postoperative day 3 . The amount of drainage was recorded after each shift. The drains were removed after the drainage volume decreased to less than $30 \mathrm{~mL} /$ day. The total volume of drainage and the number of days with a drain were recorded. All of the patients were discharged from the hospital after drain removal and were followed up weekly for 1 month. Clinically or sonographically (on routine postoperative ultrasound evaluation) detected seroma was recorded. The number of aspiration, and the total aspiration volume were also recorded.

Delayed arm motion was encouraged in all patients after surgery. Patients' followup datas were obtained from hospital charts, clinic charts, and operative reports. The data included demographics, American Society of Anesthesiologists (ASA) class, body mass index (BMI), diagnostic data, operative time and mean hospital stay. Postoperative pathologic variables including axillary lymph nodes status was also recorded.

\section{Blood collection}

Blood samples were drawn before operation and 1 hour and 24 hours after the operation. Blood samples were collected directly into serum separator tubes (Greiner, bio-one VACUETTE $\left.{ }^{\circledR}\right)$. After coagulation, centrifugation process at $1,500 \mathrm{~g}$ for $10 \mathrm{~min}$ was performed. Sera were separated, and kept frozen at $-70^{\circ} \mathrm{C}$ until analysis

\section{Measurement of ischemia-modified albumin}

Serum albumin levels (g/dL) using the colorimetry method were assayed with Advia 2400 unit (Siemens, Tarrytown, USA). The levels of serum IMA were analyzed using the rapid and colorimetric method developed by Bar et al. ${ }^{14}$ The results were reported as absorbance unit (ABSU). The adjusted IMA levels were calculated with the formula suggested by Lippi et $a l .{ }^{15}$ Albumin-adjusted IMA (AdJ-IMA) levels expressed as individual serum albumin concentration/median albumin concentration of the population $\mathrm{x}$ IMA value.

\section{Statistical analyses}

SPSS (version 15.0) was used for the statistical evaluation of the data. Categorical variables were analyzed by chisquare test; continuous variables were analyzed by the Mann-Whitney $U$ test.
Statistical significance was defined as a $\mathrm{P}$ value less than 0.05 .

\section{Results}

During the study period, 54 patients were surgically treated for breast cancer. Eighteen patients were excluded from the study. The remaining 36 patients who underwent MRM were enrolled in the study. All patients were female and had invasive ductal carcinoma of breast. In 20 of patients, dissection was performed with EC. In the remaining 16 patient's, dissection was performed with $\mathrm{PB}$. Of the 36 patients, mean age was 53.5 years (range, 40 to 78 years), mean body mass index (BMI) was $32.4 \mathrm{~kg} / \mathrm{m}^{2}$ (range, 28.1 to $39.4 \mathrm{~kg} / \mathrm{m}^{2}$ ). The comparison of patient characteristics are given in Table 1. The patients demographics, tumor stage and tumor size were similar in both groups.

There was no statistically significant difference in mean IMA levels between two groups in the preoperative period and 1 hour after operation. But, IMA levels at 24 hours after operation was higher in the $\mathrm{PB}$ group than EC group $(\mathrm{P}<0.05)$ (Table 2). Also, total wound drainage was higher in the PB group than EC group $(\mathrm{P}<0.05)$. The mean number of days with drain was found to be similar at comparison of the two groups $(\mathrm{P}>0.05)$. Patients operated with PB had more seroma aspirations than EC group, this difference was statistically significant $(\mathrm{P}<0.05)$ (Table 3). But, the total aspiration volume of seroma was not different in both groups. Intraoperative or early postoperative complication was not observed.

Table 1. Demographic and clinical data of patients.

\begin{tabular}{lccc} 
& Group EC & Group PB & P \\
No of patients & 20 & 16 & \\
Age (years) & $53.6 \pm 14.8$ & $53.5 \pm 12.8$ & NS \\
\hline BMI $\left(\mathrm{kg} / \mathrm{m}^{2}\right)$ & $31.8 \pm 4.85$ & $32.6 \pm 5.42$ & NS \\
pT2/pT3 & $14 / 6$ & $12 / 4$ & NS \\
\hline Tumor size $(\mathrm{cm})$ & $2.6 \pm 1.3$ & $2.5 \pm 1.4$ & NS \\
\hline
\end{tabular}

BMI, body mass index; PB, plasmablade; EC, electrocautery; NS, not significant. Data are presented as *mean \pm stadnard deviation.

Table 2. Adjusted ischemia modified albumin levels of patients.

\begin{tabular}{lccc} 
& $\begin{array}{c}\text { Group EC } \\
(\mathrm{n}=20)\end{array}$ & $\begin{array}{c}\text { Group PB } \\
(\mathrm{n}=16)\end{array}$ & $\mathrm{P}$ \\
Preoperative IMA level (ABSU) & $0.95 \pm 0.22$ & $0.98 \pm 0.28$ & NS \\
IMA level at 1 hour (ABSU) & $1.01 \pm 0.26$ & $1.06 \pm 0.23$ & NS \\
\hline IMA level at 24 hour (ABSU) & $1.09 \pm 0.34$ & $1.34 \pm 0.19$ & 0.034 \\
\hline
\end{tabular}

IMA, ischemia modified albumin; ABSU, absorbance unit; PB, plasmablade; EC, electrocautery; NS, not significant. Data are presented as *mean \pm stadnard deviation.

Table 3. Operative data and outcome among the groups.

\begin{tabular}{lccc} 
& $\begin{array}{c}\text { Group EC } \\
(\mathbf{n}=\mathbf{2 0})\end{array}$ & $\begin{array}{c}\text { Group PB } \\
(\mathbf{n}=\mathbf{1 6})\end{array}$ & P \\
\hline Operative time* (min) & $87.5 \pm 12.5$ & $84.6 \pm 15.3$ & NS \\
Hospital stay* (days) & $6.8 \pm 2.1$ & $7.1 \pm 1.9$ & NS \\
\hline No. of removed nodes* & $19.6 \pm 5.2$ & $18.9 \pm 4.9$ & NS \\
No. of positive nodes* & $3.2 \pm 2.4$ & $3.1 \pm 2.5$ & NS \\
\hline Drainage duration* (days) & $6.1 \pm 2.1$ & $6.5 \pm 1.6$ & NS \\
Total drainage volume* $(\mathrm{mL})$ & $233.64 \pm 107.01$ & $403.93 \pm 197.41$ & 0.012 \\
\hline Seroma & $3(15)$ & $4(25)$ & 0.015 \\
$\quad$ Number of aspirations* & $3.5 \pm 1.9$ & $4.5 \pm 1.6$ & 0.036 \\
$\quad$ Volume of aspirations* $(\mathrm{mL})$ & $116 \pm 52$ & $119 \pm 61$ & $\mathrm{NS}$ \\
Hematoma & - & - & \\
\hline
\end{tabular}

Flap necrosis

PB, plasmablade; EC, electrocautery; NS, not significant. Data are presented as *mean \pm stadnard deviation, ${ }^{*} \mathrm{n},(\%)$. 


\section{Discussion}

MRM with axillary dissection using EC is frequently associated with complications such as seroma, hematoma, prolonged axillary drainage, wound infection or necrosis, and intraoperative and postoperative bleeding. ${ }^{3-6}$ Therefore, many studies have been performed by using various electrosurgical device to reduce this complications. ${ }^{17-21}$ In addition, it is demonstrated that the use of $\mathrm{EC}$ increases the incidence of seroma formation. ${ }^{22}$ Therefore, the use of different energy sources has become widespread to prevent ischemia. Studies have been shown the efficacy of bipolar devices such as ultrasonic scissors and electrothermal bipolar vessel sealing systems to reduce the seroma formation. ${ }^{19,21}$ However, PB is a monopolar energy source, and coagulates the vessels with the heat generated by radiofrequency. The PB's working principle is similar to electrocautery. Monopolar energy sources creates high temperature to close the bleeding vessels, this heat leads to tissue ischemia and necrosis. The burned surface area increases subsequent inflammation and fluid sequestration into newly created tissue planes, which leads to seroma formation postoperatively and increases the risk of other complications, such as infection. ${ }^{22}$

Ruidiaz et al. demonstrated that the skin incisions of PB can reduce acute thermal injury depth, inflammatory response, and scar width in healing skin compared with electrosurgery. However, the effect of devices in deep tissue has not been evaluated histologically. Ruidiaz et al. reported that the clinical course of healing and time to drain removal was not different in PB and EC groups. Isik F. was addressed to this issue in a letter to the editor. ${ }^{22}$ Nevertheless, our results showed the use of PB increase the total volume of drainage, and we believe that this increase resulted from tissue ischemia. Because the IMA levels that is indicative of ischemia were significantly higher in PB group. Ultrasonic scissors and electrothermal bipolar vessel sealing systems are bipolar equipments unlike PB, hence they may reduce the axillary drainage volume via the complete closure of the lymphatic vessels. In addition, PB creates less heat in cutting area and this may also lead to not enough closure of lymphatic vessels. This is a different issue to be dealt with another prospective studies.

In the present study, we have evaluated the effect of two different energy sources on seroma formation by using a biomarker of ischemia. But, there is a limitation of this study that the choice of surgical technique to be used was based on subjective estimation of the responsible surgeon. The study groups are also not randomized. These limitations should be kept in mind while evaluating the study results.

The volume of drainage after MRM correlates with BMI, number of both total and positive nodes, and size of the tumor. ${ }^{21}$ In our study, all demographics and tumor characteristics were similar in both groups, although a significantly difference were determined in total drainage volume. This difference could be regarded as a sign of tissue ischemia.

IMA was first introduced as a serum biomarker and increase of IMA levels in patients with acute coronary syndrome has been used for an early marker of myocardial ischemia. ${ }^{23}$ However, increased IMA concentrations do not seem to depend purely on myocardial involvement. Serum IMA levels were found to be increased in non-myocardial ischemic conditions such as skeletal muscle ischemia, stroke, and pulmonary thromboembolism. ${ }^{16,24}$ The cobalt-binding capacity of albumin reduces, because of oxidative free radicals during ischemia and this new chemically changed albumin can be measured with albumin cobalt binding test. High values of IMA are closely related to ischemia. In this study, IMA values of $1^{\text {th }}$ hour were similar in both groups. However, IMA levels at 24 hours after operation was significantly high in the PB group than EC group. These findings indicates that $\mathrm{PB}$ was created more ischemia.

Shoulder dysfunction is a common complication of axillary dissection and it is necessary to mobilize the shoulder early. However, early shoulder mobilization could lead to increased seroma formation. In Shamley and colleagues systematic review of 12 randomized controlled trials showed that a delayed shoulder exercise program reduces seroma formation (odds ratio, 0.4; $95 \%$ confidence interval, 0.2-0.5; $\mathrm{P}=0.00001$ ), and no differences were found for drainage volume or hospital stay. ${ }^{25}$ Therefore, we recommend the delayed shoulder exercise program in our clinic, and all patients were subjected to delayed physiotherapy program.

The use of closed suction drainage has been a common practice to obliterate the dead space created after axillary dissection, it also decrease the incidence of wound infection, necrosis, and seroma formation. ${ }^{26}$ Patients could be discharged with drains without complications associated with early discharge or drain displacement. ${ }^{27}$ In our clinic, we used two closed suction drains one under the mastectomy flap and one in axillary space and all patients were discharged from the hospital after drain removal to keep from the concern of patients including personal care, dressing, pain and worries about the wound.

Dogan and colleagues reported that Plasmakinetic cautery reduces the drainage period and total drainage volume compared to electrocautery. But in this study, a parameter to measure the rate of ischemia has not been studied. The duration of drainage was similar to our study, although total drainage volume is very high when compared with the literature. ${ }^{19,20}$

\section{Conclusions}

The use of PB in MRM is associated with more ischemia and this situation resulted with an increased volume of total axillary drainage. PB is not a good alternative of EC for prevention of seroma formation. Although both techniques had similar complication rates, PB caused more ischemia. Prospective studies with histological evaluation are necessary to demonstrate the true result of deep tissue ischemia.

\section{References}

1. Coughlin SS, Ekwueme DU. Breast cancer as a global health concern. Cancer Epidemiol 2009;33:315-8

2. Pogson CJ, Adwani A, Ebbs SR. Seroma following breast cancer surgery. Eur J Surg Oncol 2003;29:711-7.

3. O’Dwyer PJ, O'Higgins NJ, James AG. Effect of closing dead space on incidence of seroma after mastectomy. Surg Gynecol Obstet 1991;172:55-6.

4. Vinton AL, Traverso LW, Jolly PC. Wound complications after modified radical mastectomy compared with tylectomy with axillary lymph node dissection. Am J Surg 1991;161:584-8.

5. Burak W Jr, Goodman PS, Young DC, Ferrar WB. Seroma formation following axillary dissection for breast cancer: risk factors and lack of influence of bovine thrombin. J Surg Oncol 1997;64: 27-31.

6. Lin YP, Yin WJ, Yan TT, et al. Risk factors for postoperative seromas in Chinese breast cancer patients. Chin Med J (Engl) 2011;124:1300-4.

7. Gonzalez EA, Saltzstein EC, Riedner CS, Nelson BK. Seroma formation following breast cancer surgery. Breast $\mathrm{J}$ 2003;9:385-8.

8. Hashemi E, Kaviani A, Najafi M, et al. Seroma formation after surgery for breast cancer. World J Surg Oncol 2004; 9:44.

9. Woodworth PA, McBoyle MF, Helmer $\mathrm{SD}$, Beamer RL. Seroma formation after breast cancer surgery: incidence 
and predicting factors. Am Surg 2000;66:444-50.

10. Brayant M, Baum M. Postoperative seroma following mastectomy and axillary dissection. Br J Surg 1987;74:1187.

11. Kuroi K, Shimozuma K, Taguchi T, et al. Pathophysiology of seroma in breast cancer. Breast Cancer 2005;12:288-93.

12. Porter KA, O'Connor S, Rimm E, Lopez M. Electrocautery as a factor in seroma formation following mastectomy. Am J Surg 1998;176:8-11.

13. Ruidiaz ME, Messmer D, Atmodjo DY, et al. Comparative healing of human cutaneous surgical incisions created by the PEAK PlasmaBlade, conventional electrosurgery, and a standard scalpel. Plast Reconstr Surg 2011;128:104-11.

14. Christenson RH, Duh SH, Sanhai WR, et al. Characteristics of an albumin cobalt binding test for assessment of acute coronary syndrome patients: a multicenter study. Clin Chem 2001;47:464-70.

15. Falkensammer J, Stojakovic T, Huber $\mathrm{K}$, et al. Serum levels of ischemia-modified albumin in healthy volunteers after exercise-induced calf-muscle ischemia. Clin Chem Lab Med 2007;45:535-40.

16. Turedi S, Patan T, Gunduz A, et al. Ischemia-modified albumin in the diagnosis of pulmonary embolism: an experimental study. Am J Emerg Med 2009;27:635-40.

17. Bar-Or D, Lau E, Winkler JV. A novel assay for cobalt albumin binding and its potential as a marker for myocardial ischemia: A Preliminary Report. Emerg Med J 2000;19:311-5.

18. Lippi G, Montagnana M, Salvagno GL, Guidi GC. Standardization of ischaemia modified albumin testing: adjustment for serum albumin. Clin Chem Lab Med 2007;45:261-2.

19. Manouras A, Markogiannakis H, Genetzakis $M$, et al. Modified radical mastectomy with axillary dissection using the electrothermal bipolar vessel sealing system. Arch Surg 2008;143:575-80.

20. Rohaizak M, Khan FJ, Jasmin JS, et al. Ultracision versus electrocautery in performing modified radical mastectomy and axillary lymph node dissection for breast cancer: a prospective randomized control trial. Med J Malaysia 2013;68:204-7.

21. Lumachi F, Brandes AA, Burelli P, et al. Seroma prevention following axillary dissection in patients with breast cancer by using ultrasound scissors: a prospective clinical study. Eur J Surg Oncol 2004;30:526-30.

22. Isik F. Discussion: Comparative healing of human cutaneous surgical incisions created by the PEAK PlasmaBlade, conventional electrosurgery, and a standard scalpel. Plast Reconstr Surg 2011;128:112-3.

23. Christenson RH, Duh SH, Sanhai WR, et al. Characteristics of an albumin cobalt binding test for assessment of acute coronary syndrome patients: a multicenter study. Clin Chem 2001;47:464-70.

24. Falkensammer J, Stojakovic T, Huber $\mathrm{K}$, et al. Serum levels of ischemia-modified albumin in healthy volunteers after exercise-induced calf-muscle ischemia. Clin Chem Lab Med 2007;45:535-40.

25. Shamley DR, Barker K, Simonite V, Beardshaw A. Delayed versus immediate exercises following surgery for breast cancer: a systematic review. Breast Cancer Res Treat 2005;90:26371.

26. Divino CM, Kuerer HM, Tartter PI. Drains prevent seromas following lumpectomy with axillary dissection. Breast J 2000;6:31-3.

27. Holcombe C, West N, Mansel RE, Horgan K. The satisfaction and savings of early discharge with drain in situ following axillary lymphadenectomy in the treatment of breast cancer. Eur J Surg Oncol 1995;21:604-6. 